\title{
Radiobiologie avec des neutrons rapides
}

\author{
C. DIONET ${ }^{1}$, A. TCHIRKOV ${ }^{1}$, J. ARNOLD ${ }^{1}$, Z. FRANCIS ${ }^{1}$, J.-P. ALARD ${ }^{1}$, \\ M. RAPP ${ }^{1}$, P. VERRELLE ${ }^{1}$
}

(Manuscrit reçu le 6 novembre 2006, accepté le 28 février 2007)

RÉSUMÉ Des cellules d'un cancer humain (mélanome) particulièrement résistant à des doses classiques de radiothérapie médicale (rayons $X$ de haute énergie) ont été irradiées avec des neutrons de $14 \mathrm{MeV}$ à des doses étagées de 5 centiGrays (cGy) jusqu'à 1,12 Gray $(\mathrm{Gy})$, à deux débits de doses, l'un très faible $\left(0,8 \mathrm{mGy} \mathrm{mn} \mathrm{mb}^{-1}\right)$, l'autre modéré (40 mGy mn $\left.{ }^{-1}\right)$. L'effet biologique a été étudié par deux techniques : analyse de la survie cellulaire et des anomalies chromosomiques. Un aspect inhabituel de la forme de la courbe de survie a été mis en évidence au très faible débit de dose : mort cellulaire aiguë à 5 cGy suivie d'un plateau de survie de 10 à 32,5 cGy. Le taux d'aberrations chromosomiques induites suit lui-même un plateau au très faible débit de dose, de 10 à 30 cGy. Ces phénomènes disparaissent à plus fort débit de dose, ce qui suggère l'existence d'un seuil de déclenchement de la réparation cellulaire et une saturation vers $35 \mathrm{cGy}$. Ces résultats ont un intérêt pour la radiobiologie des neutrons et la radioprotection.

ABSTRACT Radiobiology with fast neutrons.

Human melanoma cells particularly resistant to high energy $X$ rays were irradiated with $14 \mathrm{MeV}$ neutrons at low doses ranging from 5 cGy to $1.12 \mathrm{~Gy}$ and using two different dose rates: a very low one $\left(0.8 \mathrm{mGy} \mathrm{mn}^{-1}\right)$ and a moderate one $\left(40 \mathrm{mGy} \mathrm{mn}^{-1}\right)$. The biological effects of neutrons were studied both by cell survival assay and chromosomal aberrations analysis. Unusual features of the survival curve at low dose rate were observed as a marked degree of cell killing at $5 \mathrm{cGy}$ followed by a plateau of survival from 10 to $32.5 \mathrm{cGy}$. At low dose rate, the levels of induced chromosomal aberrations tended also to reach a plateau with doses ranging from 10 to $30 \mathrm{cGy}$. Both phenomena disappear at higher dose rate. The existence of a plateau observed after low-dose neutrons suggests that a repair process might be induced after a threshold dose of 5-7.5 cGy with a saturation around $32.5 \mathrm{cGy}$. These findings may be of interest for the domain of neutrons radiobiology and radioprotection.

Keywords: neutrons / radiobiology / low dose rate / radioprotection / computing grid / Monte Carlo simulation / cells repair process

\section{Introduction}

La radiophysique et la dosimétrie constituent le thème de recherche de trois des équipes du Laboratoire de Physique Corpusculaire (LPC). Les axes de recherche en sont cependant très différents.

L'équipe PCSV du LPC (Thiam, Maigne, Breton, Donnarieix) effectue des simulations Monte Carlo des dépôts de doses en radiothérapie, curiethérapie et

\footnotetext{
${ }^{1}$ Laboratoire de Physique Corpusculaire, 24 avenue des Landais, 63177 Aubière Cedex, France.
} 
déploiement sur grille de calcul. La méthode de calcul Monte Carlo est reconnue aujourd'hui comme l'algorithme pouvant modéliser au plus près les phénomènes physiques liés aux dépôts d'énergie dans un milieu. D'où l'intérêt d'utiliser cette méthode dans la planification du traitement du cancer par radiothérapie, les systèmes de planification traitement (TPS) existants étant limités dans la précision des calculs pour certains cas bien spécifiques. Dans cette approche, l'équipe PCSV s'intéresse à la validation du code de calcul Monte Carlo GATE pour les applications dosimétriques en physique médicale. Cependant, l'utilisation de la méthode Monte Carlo pour des géométries complexes ou des images de haute résolution nécessite de nombreuses heures de calculs. En effet, un résultat précis ne peut être obtenu qu'en générant beaucoup d'événements. L'équipe PCSV étudie donc les capacités de la grille de calcul notamment en termes de réduction $\mathrm{du}$ temps de calcul pour déployer les simulations avec GATE sur un environnement de calcul distribué.

L'équipe thermoluminescence du LPC (Bassinet, Courtine, Miallier, Pilleyre, Sanzelle) met en œuvre et développe des techniques de mesure des faibles radioactivités dans deux directions : mesures de l'activité gamma de matériaux solides ou liquides par spectroscopie gamma avec un détecteur germanium; dosimétrie in situ du débit de dose d'irradiation, dans des milieux naturels, par thermoluminescence, ou avec une sonde à scintillation.

Cette activité est initialement liée à une application précise, à savoir la mesure de la radioactivité naturelle dans le cadre de la datation par thermoluminescence en archéologie et en volcanologie. Les contraintes et exigences spécifiques de ce contexte, notamment un bas niveau de radioactivité et la multiplicité des sources, ont induit l'acquisition de méthodes et compétences qui sont régulièrement mises à profit en dehors de la datation: suivi de la radioactivité atmosphérique (collaboration ATMO Auvergne), expertises à la demande (sociétés privées, institutionnels) pour des mesures de faibles activités, naturelles ou artificielles. Ces compétences ont été validées par des Certificats de Qualification Technique délivrés par l'OPRI (arrêté du 22/05/98). Récemment, une étape importante a été franchie pour obtenir un agrément au titre du réseau national de mesures de la radioactivité de l'environnement (arrêté du 17 octobre 2003) : la participation avec succès à la campagne 2005 d'inter-comparaisons, organisées par l'IRSN, portant sur la mesure de radioéléments émetteurs gamma naturels (sédiment de lac $\mathrm{N}^{\circ} 77$ SR 300) ou artificiels (eau rechargée $\mathrm{N}^{\circ} 76$ EE 300).

L'équipe neutrons a pour thématique la radiobiologie des neutrons rapides. C'est cet axe de recherche que nous allons développer dans ce numéro spécial.

Depuis de nombreuses années, les neutrons rapides font l'objet de controverses quant à leur efficacité biologique relative (EBR) aux faibles doses (Worgul et al., 
1996). De plus, il existait un dogme accréditant l'idée que le débit de dose des neutrons n'avait pas d'influence sur leur pouvoir cytotoxique (Elkind, 1991). La courbe de survie de cellules tumorales irradiées par des neutrons apparaît classiquement comme une droite sur une échelle semi-logarithmique (doses en cGy en abscisses, survie en ordonnées logarithmiques). Les courbes de survie de cellules irradiées en photons ou rayons X comportent un «épaulement» initial correspondant à la réparation de dommages sublétaux. Il a été montré que des cellules radiorésistantes pouvaient manifester une hypersensibilité à de faibles doses de rayons X (Lambin et al., 1993). Il était admis que les neutrons ne créaient pas ou peu de lésions sublétales, expliquant ainsi l'absence d'épaulement des courbes de survie. Cependant, très peu de résultats concernent les neutrons délivrés à très faibles doses et très faible débit de dose. De même, les aberrations chromosomiques engendrées par les neutrons sont sensées suivre une courbe linéaire (Revell, 1983).

D'autre part, l'emploi de neutrons en thérapeutique anti-tumorale humaine a fait apparaître d'importantes séquelles sur les tissus sains, laissant penser qu'ils sont peut-être utilisés à des doses trop importantes. Ces réflexions nous ont conduits à explorer l'effet des neutrons, délivrés à très faibles doses et débit de dose, sur des cellules d'un cancer humain particulièrement agressif et radiorésistant, le mélanome.

\section{Matériel et méthodes}

\subsection{Culture cellulaire et étude cytogénétique}

Une lignée cellulaire d'un mélanome humain (M4 Beu) est cultivée dans un milieu d'Eagle et maintenue en incubateur à $37{ }^{\circ} \mathrm{C}$ avec $5 \%$ de $\mathrm{CO}_{2}$ en atmosphère humide. Pour les expérimentations, les cellules sont ensemencées la veille dans des flacons corning de $25 \mathrm{~cm}^{2}$. Après irradiation, les flacons sont placés à l'incubateur et les colonies engendrées par les cellules survivantes comptées au terme de 2 semaines.

Les études chromosomiques sont réalisées sur des cellules ayant atteint la mitose au cours de la première division après irradiation. Les aberrations chromosomiques spontanées ont été appréciées sur 172 cellules non irradiées. La fréquence et le type d'aberrations après irradiation ont été mesurés en examinant entre 80 et 104 cellules pour le débit de dose modéré et entre 295 et 311 cellules au très faible débit de dose.

\subsection{Irradiation en neutrons à très faible débit de dose}

Le très faible débit de dose provient de la réaction $\mathrm{D}\left(\mathrm{T},{ }^{3} \mathrm{He}\right)$ n d'un générateur scellé. Les neutrons de $14 \mathrm{MeV}$ sont émis à un flux de $3 \times 10^{8}$ neutrons. ${ }^{-1}$. La dose 
délivrée aux flacons de culture est mesurée par des diodes, le flux de neutrons contrôlé par un compteur ${ }^{3} \mathrm{He}$. La dosimétrie est assurée par des chambres d'ionisation, un compteur Geiger-Muller, un scintillateur BGO, des chambres de fission, des diodes au silicium PIN. Une chambre d'ionisation argon et un compteur Geiger-Muller protégé par une enveloppe de lithium permettent de mesurer la dose de rayons $X$. Le débit de dose mesuré est de $0,8 \mathrm{mGy} \mathrm{m}^{-1}( \pm 5 \%)$ pour les neutrons et de $0,07 \mathrm{mGy} \mathrm{mn}^{-1}( \pm 15 \%)$ pour les rayons «X $»$. Le pic de neutrons de $14 \mathrm{MeV}$ est prédominant à $98 \%$.

\subsection{Irradiation en neutrons à débit de dose modéré}

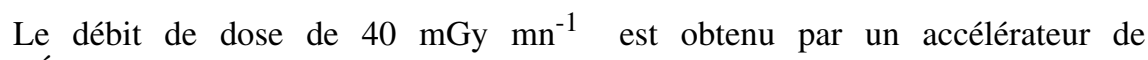
l'Établissement Technique Central de l'Armement (ETCA) délivrant un flux de $5 \times 10^{11}$ neutrons s${ }^{-1}$.

\subsection{Irradiation par rayons $X$}

Les cellules sont irradiées à l'équilibre électronique par un accélérateur médical produisant des photons de $6 \mathrm{MV}$, au débit de dose utilisé en thérapeutique humaine de $3 \mathrm{~Gy} \mathrm{mn}^{-1}$.

\subsection{Contrôle de température}

Toutes les expérimentations sont réalisées à $37^{\circ} \mathrm{C}$ grâce à un système électronique entourant les flacons de culture.

\section{Résultats et discussion}

La figure 1 rapporte le détail des survies cellulaires. La courbe obtenue au très faible débit de dose présente trois phases : (1) une pente initiale très marquée ; (2) un plateau de survie de $65 \%$ depuis 5 cGy jusqu'à environ 32,5 cGy ; (3) une décroissance monotone habituellement rapportée après irradiation en neutrons. Le test de Kruskal-Wallis ne trouve pas de différence significative dans la survie cellulaire de 5 à 32,5 cGy. Au débit de dose plus fort, la mort cellulaire aiguë initiale et le plateau de survie disparaissent pour laisser place à une décroissance sensiblement exponentielle. La différence entre les deux courbes est significative pour les survies comprises entre 5 cGy et 20 cGy par le test de MANOVA ( $\mathrm{p}<$ $0,001)$ et par le modèle de régression des moindres carrés.

La figure 2 montre les différences d'aspect entre les courbes de survie cellulaire après irradiation par rayons $\mathrm{X}(6 \mathrm{MV})$ et neutrons à très faible débit de dose. Les cellules sont peu sensibles aux rayons $\mathrm{X}$ : une dose de 175 cGy est 


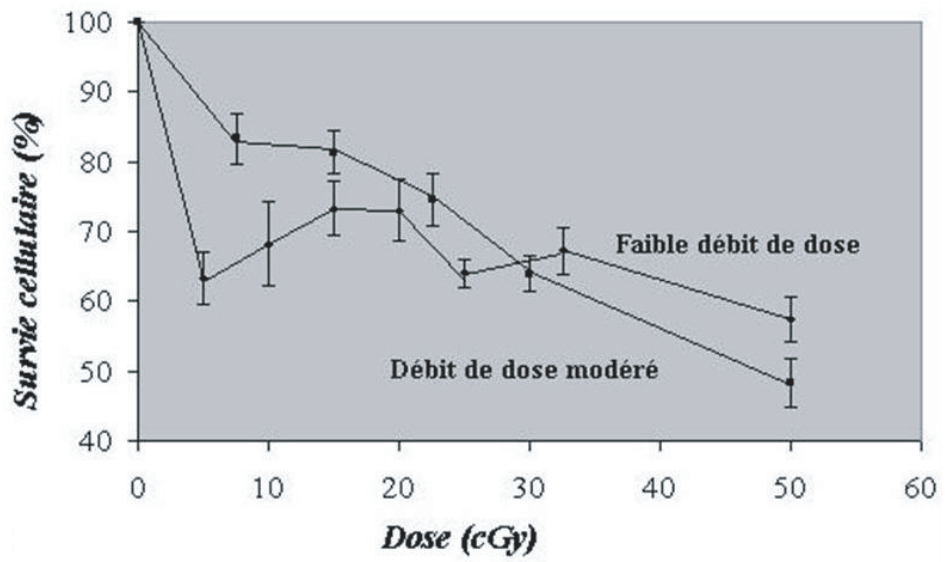

Figure 1 - Survie cellulaire à faible débit de dose et débit de dose modéré.

Cell survival curves obtained after neutron irradiation of melanoma, cell line at low and moderate dose rates.

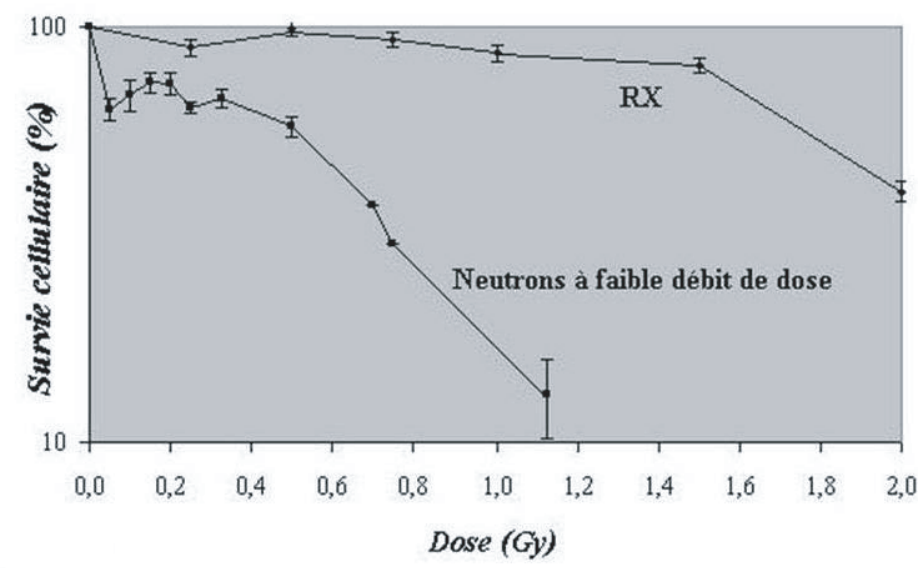

Figure 2 - Survie cellulaire après irradiation par rayons $X$ et neutrons à faible débit de dose.

Survival curves for melanoma cells after $X$ and low dose rate neutron irradiation.

nécessaire pour que la survie chute à $65 \%$, taux de survie atteint avec seulement 5 cGy en neutrons. L'efficacité biologique relative des neutrons (EBR) est le rapport de la dose de rayons $\mathrm{X}$ sur la dose de neutrons produisant le même effet biologique. Si l'on considère l'EBR à la survie cellulaire de $65 \%$, on constate que 

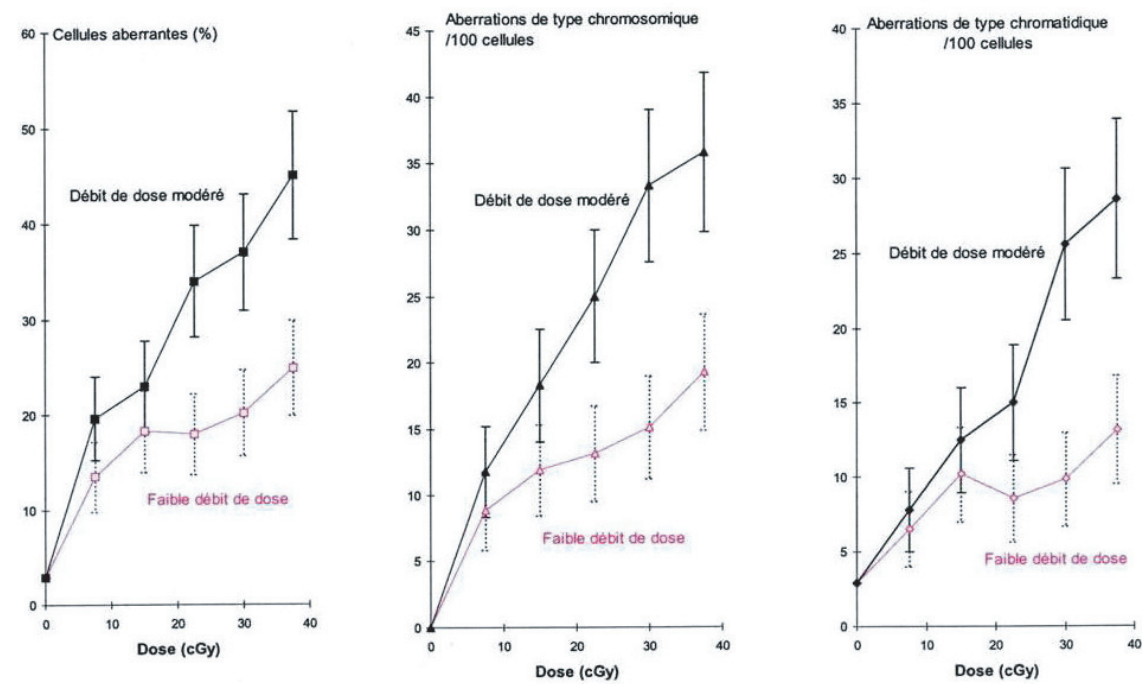

Figure 3-Courbes dose-réponses des diverses aberrations cellulaires, chromosomiques et chromatidiques.

Dose response curves for the induction of aberrant cells and chromatid aberrations in melanoma cells by low and moderate dose rate neutrons.

cette survie nécessite 175 cGy de rayons X, et correspond à une gamme de doses neutrons allant de 5 à 32,5 cGy. De ce fait, l'EBR des neutrons, à ce niveau de survie, varie environ de 35 à 5.

La figure 3 représente les courbes dose-réponses des diverses aberrations cellulaires, chromosomiques et chromatidiques obtenues après irradiation par neutrons à très faible débit de dose, et débit de dose modéré. Avec le débit de dose modéré, on constate une croissance linéaire des aberrations, alors qu' au très faible débit de dose les aberrations ont une tendance à s'infléchir en plateau.

\section{Simulation des interactions des neutrons rapides avec le milieu environnant}

Afin de comprendre et de pouvoir analyser les conditions dans lesquelles les cultures cellulaires sont irradiées, on effectue des simulations basées sur la méthode Monte Carlo. Les simulations permettent d'étudier les interactions des neutrons rapides avec le milieu environnant et le milieu de culture cellulaire, afin d'une part de donner une idée détaillée sur les particules qui atteignent la cellule 


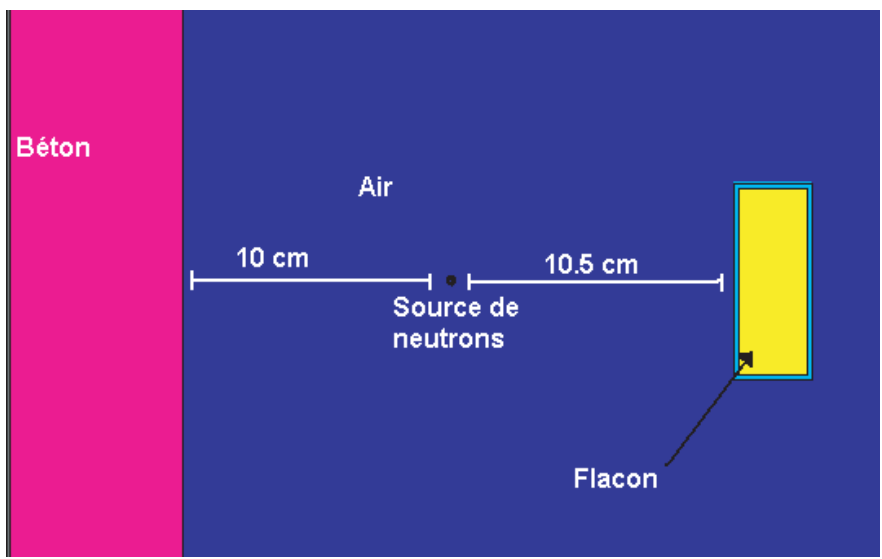

Figure 4 - Géométrie modélisée dans les simulations.

Geometry model for simulations.

lors des irradiations et d'autre part d'estimer la dose déposée par ces particules dans la cellule. La casemate en béton a été prise en compte avec une source isotropique émettant des neutrons de $14 \mathrm{MeV}$ d'énergie placée à l'intérieur de cette casemate. À $10,5 \mathrm{~cm}$ de la source est placé un flacon en PMMA rempli d'eau. À l'intérieur du flacon est définie une sphère en eau de 30 micromètres de diamètre. L'eau étant le constituant majeur du corps humain, il est donc possible de considérer que la cellule est remplie d'eau. Ceci nous permet d'estimer la dose déposée au niveau de la cellule durant les irradiations. La composition du béton utilisé est la suivante :

$\begin{array}{ll}\text { hydrogène } & 1,080 \% \text { (pourcentage en masse), } \\ \text { oxygène } & 57,180 \%, \\ \text { aluminium } & 3,675 \%, \\ \text { silicium } & 36,430 \%, \\ \text { fer } & 1,510 \%, \\ \text { carbone } & 0,108 \%,\end{array}$

la composition du PMMA est la suivante :

$\begin{array}{ll}\text { hydrogène } & 8,050 \%, \\ \text { carbone } & 60,000 \%, \\ \text { oxygène } & 31,950 \% .\end{array}$

La figure 4 représente la configuration modélisée dans les simulations. 


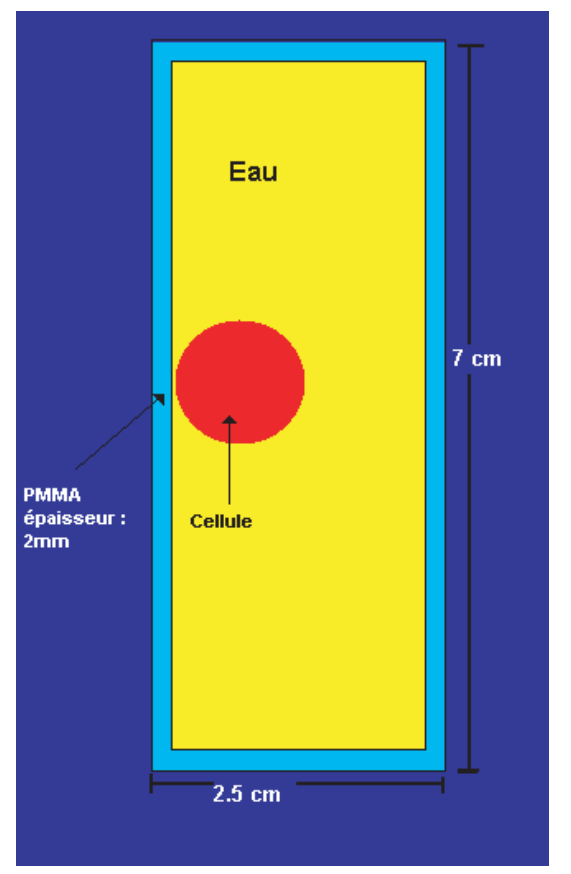

Figure 5-Géométrie du flacon contenant les cellules irradiées et position d'une cellule dans le flacon (échelle non respectée).

Geometry of a flasket containing the irradiated cells and position of a cell in the flasket.

La figure 5 montre le flacon dans lequel est placée la cellule (échelle arbitraire) ainsi que la position de la cellule dans le flacon.

La figure 6 montre le flux des neutrons qui traverse la face interne du PMMA contre laquelle est collée la cellule.

Les neutrons ayant une énergie supérieure à $14 \mathrm{MeV}$ sont les neutrons provenant directement de la source. La composante dégradée du spectre obtenu est due aux diffusions diverses des neutrons contre les murs de la casemate. On remarque que presque $10 \%$ des neutrons qui atteignent le milieu cellulaire ont une énergie inférieure à $1 \mathrm{MeV}$.

La dose déposée au niveau de la cellule placée à $10,7 \mathrm{~cm}$ de la source est de $4,905 \mathrm{cGy} \mathrm{h}^{-1}$, sachant que l'erreur relative du résultat est inférieure à $1 \%$. Le résultat obtenu expérimentalement à $10,5 \mathrm{~cm}$ est de $5 \mathrm{cGy} \mathrm{h}^{-1}$ ( $2 \%$ d'erreur).

Des simulations plus détaillées sont actuellement développées ; elles tiennent compte des particules secondaires créées dans le milieu cellulaire comme les protons, les électrons. 


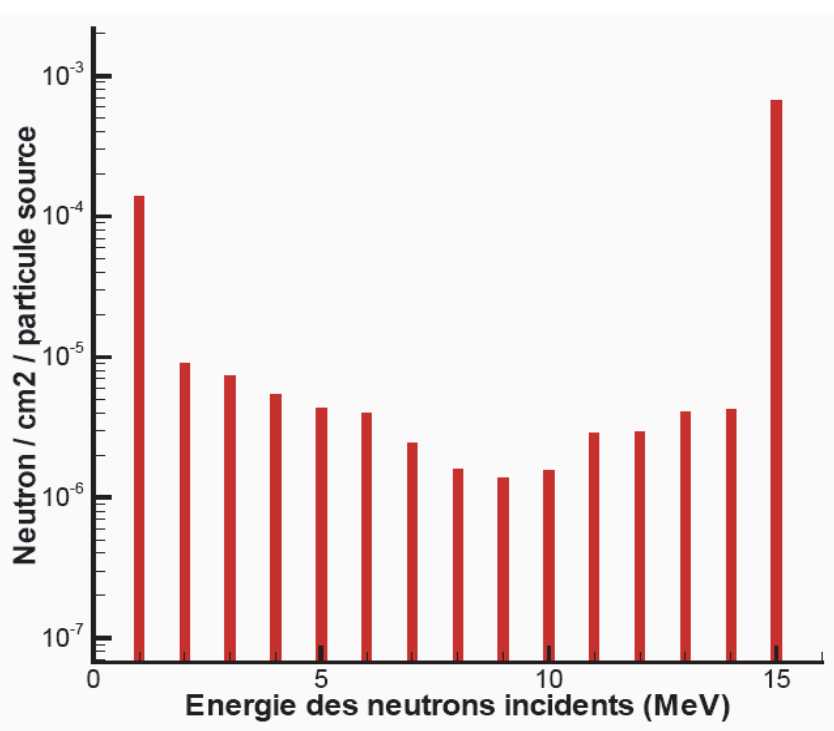

Figure 6 - Flux de neutrons entrant dans le flacon à travers la surface contre laquelle est collée la cellule. Neutrons flow coming into the flasket through the surface against which the cell is stuck.

\section{Conclusion et perspectives}

Par deux méthodes différentes, courbes de survie cellulaire et analyse des anomalies cytogénétiques, nous avons montré que sur un cancer radiorésistant, de très faibles doses de neutrons avaient un pouvoir tumoricide important, à condition que le débit de dose soit très faible. L'hypothèse avancée est qu'il existe un seuil de déclenchement de la réparation cellulaire en dessous duquel la cellule ne peut réagir et meurt. Lorsque la dose augmente, les processus de réparation se mettent en route, expliquant la constance de la survie jusqu'aux environ de $35 \mathrm{cGy}$. Audelà de cette dose les systèmes enzymatiques de réparation sont saturés et la mort cellulaire s'accroît de façon monotone. Il s'agit là de la première démonstration patente de l'existence d'un phénomène non linéaire dans la survie cellulaire aux faibles doses de neutrons et du rôle du débit de dose. Les études cytogénétiques corroborent l'idée qu'une réparation est possible au cours de l'irradiation neutronique. L'existence d'un plateau de survie entre 5 et 32,5 cGy peut expliquer les divergences rencontrées dans les publications concernant la valeur de l'EBR à très faibles doses de neutrons (Dionet et al., 2000). Ces travaux ont conduit à une collaboration étroite entre radiobiologistes et physiciens débouchant sur l'élaboration de codes de simulation (Alard et al., 2002) dont nous avons reporté 
ci-dessus les principales lignes. Les études en cours portent sur l'analyse d'autres lignées cellulaires et sur la quantification du seuil de déclenchement de la réparation cellulaire. Les résultats obtenus contribuent à porter un regard nouveau sur l'effet biologique des neutrons à très faibles doses et débit de dose. Ils ouvrent des discussions sur les modalités d'utilisation de la neutronthérapie, et sur les éventuels risques environnementaux dans les milieux où règnent de faibles doses de neutrons : vols en altitude, accélérateurs de particules.

\section{RÉFÉRENCES}

Alard J.-P., Bodez V., Tchirkov A., Arnold J., Nénot M.L., Crespin S., Rapp M., Verrelle P., Dionet C. (2002) Simulation of neutron interactions at the single-cell level, Rad. Res. 158, 650-656.

Dionet C., Tchirkov A., Alard J.-P., Arnold J., Dhermain J., Rapp M., Bodez V., Tamain J.-C., Monbel I., Malet P., Kwiatkowsky F., Donnarieix D., Veyre A., Verrelle P. (2000) Effects of low-dose neutrons applied at reduced dose rate on human melanoma cells, Rad. Res. 154, 406-411.

Elkind M.M. (1991) Physical, biophysical and cell-biological factors that can contribute to enhanced neoplastic transformation by fission-spectrum neutrons, Rad. Res. 128 (Suppl.), 47-52.

Lambin P., Marples B., Fertil B., Malaise E.P., Joiner M.C. (1993) Hypersensitivity of a human tumor cell line to very low radiation doses, Int. J. Radiat. Biol. 63, 639-650.

Revel S.H. (1983) Relationship between chromosome damage and cell death, Radiation-induced chromosome damage in man (T. Ishihara and M.S. Sasaki, Eds) pp. 215-233. Alan R. Lis, New York.

Worgul B.V., Medvedovsky C., Huang Y., Marino S.A., Randers-Pehrson G., Brenner D.J. (1996) Quantitative assessment of the cataractogenic potential of very low doses of neutrons, Rad. Res. 145, 343-349. 